\title{
A Prospective Survey Comparing Patients' Experience of a Nurse-Led Clinic and Consultant-Led Clinic for Surveillance of Patients with Dilatation of the Intrathoracic Aorta
}

\author{
Bhuvaneswari Krishnamoorthy1,2,3*, Rashmi Birla1, William R. Critchley4, Paul D. Waterworth1 \\ ${ }^{1}$ Department of Cardiothoracic Surgery, Manchester Foundation NHS Trust, Manchester, UK \\ ${ }^{2}$ Division of Cardiovascular Sciences, Faculty of Biology, Medicine and Health, The University of Manchester, Manchester, UK \\ ${ }^{3}$ Faculty of Health and Social Care, Edge Hill University, Ormskirk, UK \\ ${ }^{4}$ The Endothelial Cell Biology Unit, School of Molecular and Cellular Biology, University of Leeds, Leeds, UK \\ Email: *bhuvaneswari.bibleraaj@mft.nhs.uk
}

How to cite this paper: Krishnamoorthy, B., Birla, R., Critchley, W.R. and Waterworth, P.D. (2019) A Prospective Survey Comparing Patients' Experience of a Nurse-Led Clinic and Consultant-Led Clinic for Surveillance of Patients with Dilatation of the Intrathoracic Aorta. World Journal of Cardiovascular Surgery, 9, 24-34. https://doi.org/10.4236/wjcs.2019.92003

Received: June 25, 2018

Accepted: February 25, 2019

Published: February 28, 2019

Copyright $\odot 2019$ by author(s) and Scientific Research Publishing Inc. This work is licensed under the Creative Commons Attribution International License (CC BY 4.0).

http://creativecommons.org/licenses/by/4.0/

\section{(c) (i) Open Access}

\begin{abstract}
Background: Increasing workload in consultant-led clinics often means patients to wait a long time for clinic appointments. To address this, there is an increasing trend in developing nurse-led clinics across many specialities in the National Health Service. This study aims to assess whether the implementation of a nurse-led clinic in thoracic aortic surgery will optimise the utilisation of health care services and improve overall patient satisfaction. Methods: 80 follow-up patients were asked to complete a questionnaire following their appointment in an aortic clinic, which was led either by a consultant $(n=40)$ or an aortic specialist nurse $(n=40)$. All patients seen by a nurse in the clinic were assessed by a consultant surgeon prior to the clinic for suitability. No new patients were seen by a nurse. Any patient with an aortic dimension of $5 \mathrm{~cm}$ or greater was seen by the consultant. If there were any complicated clinical features, the patient was seen in the consultant-led clinic. Patients were asked questions about their time spent with the respective health care professionals across 12 categories (punctuality, preparedness, understanding of concerns, clarity of speech, listening, respect, explaining, letting you talk, putting you at ease, emotional support, advice and advice for next follow-up). Patients rated each category using an ordinal scale from 0 10. Results: Patient scores were greater in nurse-led clinics compared to consultant-led clinics across a number of categories although only punctuality reached significance (mean 9.2 vs. $6.8, \mathrm{p}<0.001$ ). Scores for preparedness
\end{abstract}


(mean 9.5 vs. 9.0, $\mathrm{p}=0.29$ ) and understanding of worries (mean 9.8 vs. 9.5, $\mathrm{p}$ $=0.18$ ) were higher in nurse-led clinics but were not significant. Similar scores for both nurse and consultant-led clinics were recorded for the remaining categories: $(\mathrm{p}>0.05)$. Conclusion: Patients were highly satisfied with the nurse-led clinic across all categories, with greater satisfaction for punctuality. These findings suggest that a nurse-led clinic can be implemented for the management of carefully selected thoracic aortic surgery patient without reduction in patient satisfaction.

\section{Keywords}

Nurse-Led Clinic, Consultant-Led Clinic, Thoracic Aortic Surgery, Surveillance, Patient Satisfaction

\section{Introduction}

Intrathoracic aortic dilatation is often clinically silent and can be fatal to patients. Continuous pre-operative monitoring and surveillance of these patients is vital. Many nurses have developed advanced roles, previously carried out by medical professionals. Nurse-led clinics have been promoted in different medical specialities in order to reduce the outpatient clinic waiting times [1] and running cost without compromising the standard of care [2]. The introduction of a follow-up nurse-led clinic in aortic surgery may provide additional time for consultant surgeons to assess new or higher risk patients rather than running routine follow-up clinics.

Nurse-led clinics are well established in general surgery [3], primary care [4], and orthopaedic surgery and in wound assessment clinics [5]. Nurse-led clinics for the management of patients with intrathoracic aortic dilatation surgery do not exist in the UK. The aim of this prospective study was to assess the patient's experiences in nurse-led and consultant-led clinics for surveillance of patients with intrathoracic aortic dilatation.

The nurse-led clinics are not simply replacing the consultants with a nurse. The nurse-led clinics can enhance the patient quality of care, wellbeing and also provide better two-way communication [6]. Good communication and continuity of care are one of the main factors for patient dissatisfaction in addition to outpatient waiting time, duration of consultations [7]. Getting and access to coherent information about the surgery is a prerequisite for making informed decisions. In addition, good consultation in the outpatient clinic reduces patient anxiety, stress level and can lead to better quality of life [8] [9].

Cancer care has demonstrated better consultation and improvement in patient outcomes in nurse-led clinics [10] [11]. The nurse-led clinic not only provides just an assessment of the patient. It provides more holistic care, support, education for the families and patients [7]. However, still there is a paucity of nurse-led clinic exploratory studies in surgery. Patient education promotes 
self-care, treatment adherence and supports the patient to cope more effectively with their illness [12]. To educate the patient it needs time and more effort to simply the facts to their level. The consultants do not have time to provide this due to the complexity of their work-load in the National Health Service.

\section{Methods}

This prospective observational survey was conducted in University Hospital of South Manchester NHS Foundation Trust Cardiac Surgery centre from January 2015 to June 2015. This audit was approved by the hospital audit department and all the patients provided verbal consent before participating in this audit. A total of 86 patients were recruited and asked to complete a questionnaire for this study. Group 1 consists of 42 patients who attended the consultant-led aortic surgery clinic and Group 2 consists of 44 patients who attended the aortic surgery nurse-led clinic. However, a total of six patients across the two groups were excluded because the data collection form was in-complete.

All the patients seen by the nurse in the clinic were assessed by the consultant surgeon for suitability. The nurse saw only routine follow-up patients who had an aortic dimension less than $5 \mathrm{cms}$. All new patients or follow-up patients with an aortic dimension of $5 \mathrm{cms}$ or greater were seen by the consultant. The responsibilities of the specialist nurse practitioner in the clinic were as follows: review of the aortic scan (CT or MRI) prior to the clinic, routine check-up of the patients, discussion of the scan results with patients and families, health education to answer any questions raised by the patients and to give advice until the next follow-up. The consultant was available to address any issues or complicated clinical features diagnosed during consultation.

The aortic specialist nurse had undergone a period of careful training by the consultant surgeon to ensure that she was able to safely assess patients, interpret scan results and give advice regarding management. Training also involved the ability to recognise when a change in the size of the aneurysm represented a significant increase and when to refer the patient back to the consultant surgeon.

All patients were asked to complete a specific questionnaire (Appendix 1) anonymously and asked to put it in an envelope and seal it before handing over to the clinic nurse manager. They were asked with regard to their time spent with the respective health care professionals across 12 categories (punctuality, preparedness, understanding of concerns, clarity of speech, listening, respect, explaining, letting you talk, putting you at ease, emotional support, advice and advice for next follow-up) with particular focus on the explanation they received about the surgical procedure. Patients rated each category using an ordinal scale from 0 - 10. The answers from the questionnaires were entered and results were collected and evaluated by an independent practitioner who did not participate in the clinics.

\section{Sample Calculation and Data Analysis}

Due to lack of studies in this area in surgical speciality. This study was con- 
ducted as an observational prospective survey. We used small, convenience sampling process for this study. The patient sample size was estimated and selected according to the process-based audit which is accepted to get a "snapshot" roughly 20 - 50 cases [13]. Choosing a larger sample size than is necessary which takes up extra time and resources without adding any value.

The distribution of data was assessed using the Shapiro Wilk test. Subsequently, all parameters were analysed for statistical significance using the Mann Whitney $U$ test. Statistical significance was accepted if $\mathrm{p}<0.05$. All analysis was performed with Prism v7.01 (GraphPad, San Diego, CA, USA).

\section{Results}

Clinics led by nurses scored significantly higher in terms of punctuality of appointments compared with consultant-led clinics (nurse-led vs. consultant-led: $9.3 \pm 1.5$ vs. $6.8 \pm 3.3, \mathrm{p}<0.0001$, Figure 1$)$. However, no other differences were established between any of the other factors surveyed in this study. Indeed, similar scores were observed for preparedness (nurse-led vs. consultant-led: $9.5 \pm$ 1.6 vs. $9.0 \pm 2.3, \mathrm{p}=0.44$, Figure $2(\mathrm{a})$ ), understanding of patient concerns (nurse-led vs. consultant-led: $9.8 \pm 0.6$ vs. $9.5 \pm 1.7, \mathrm{p}=0.28$, Figure $2(\mathrm{~b})$ ) and clarity of speech (nurse-led vs. consultant-led: $9.8 \pm 0.6$ vs. $9.5 \pm 1.7, \mathrm{p}=0.28$, Figure 2(c)).

Patient scores recorded for listening were generally high in both groups, with no differences observed between nurse and consultant-led clinics (nurse-led vs. consultant-led: $9.8 \pm 0.6$ vs. $9.7 \pm 1.3, p=0.69$, Figure $3(a)$ ). A similar pattern was observed for level of respect (nurse-led vs. consultant-led: $9.9 \pm 0.4$ vs. $9.7 \pm$ $1.4, \mathrm{p}=0.92$, Figure $3(\mathrm{~b})$ ), quality of explanation (nurse-led vs. consultant-led: $9.9 \pm 0.5$ vs. $9.7 \pm 1.5, \mathrm{p}=0.90$, Figure $3(\mathrm{c}))$, and letting the patient speak (nurse-led vs. consultant-led: $9.9 \pm 0.5$ vs. $9.5 \pm 1.6, \mathrm{p}=0.79$, Figure $3(\mathrm{~d})$ ).

Patients reported high scores for being put at ease by both nurses and consultants during the clinics (nurse-led vs. consultant-led: $9.9 \pm 0.4$ vs. $9.6 \pm 1.6, \mathrm{p}=$ 0.79 , Figure $4(\mathrm{a})$ ). Lower scores were provided for the level of emotional support given in both groups, with no differences observed dependent upon the type of clinic (nurse-led vs. consultant-led: $8.5 \pm 3.2$ vs. $8.1 \pm 3.5$, p $=0.64$, Figure 4(b)). The patients' scores were consistently high for advice (nurse-led vs. consultant-led: $9.3 \pm 2.0$ vs. $9.2 \pm 2.3, \mathrm{p}=0.92$, Figure $4(\mathrm{c}))$ and advice for the next follow-up visit (nurse-led vs. consultant-led: $9.6 \pm 1.5$ vs. $9.5 \pm 1.9, \mathrm{p}=0.93$, Figure $4(d)$ ) regardless of the type of clinic.

\section{Discussion}

According to our knowledge this is the first observational study evaluating patient perceptions and reported satisfaction levels of intrathoracic aortic aneurysm clinics led by either nurses or consultants. Many studies have highlighted that nurse-led clinics are patient-friendly [1], reduce waiting times [3] and provide more emotional support and higher satisfaction for the patient [14]. Our 


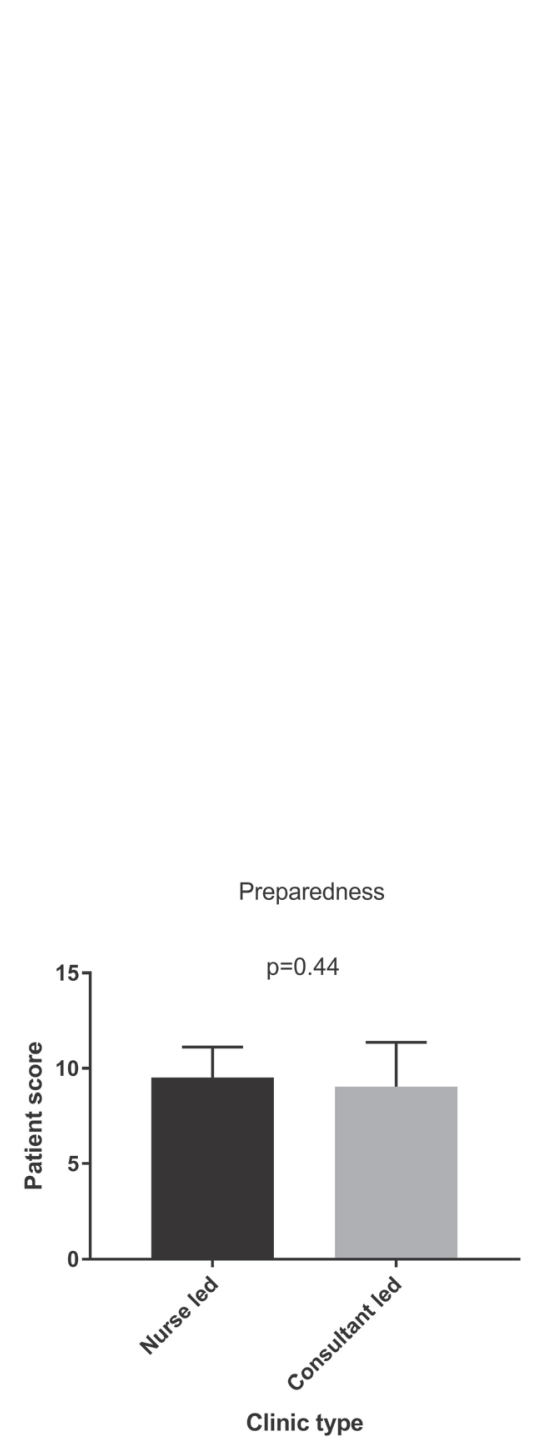

(a)

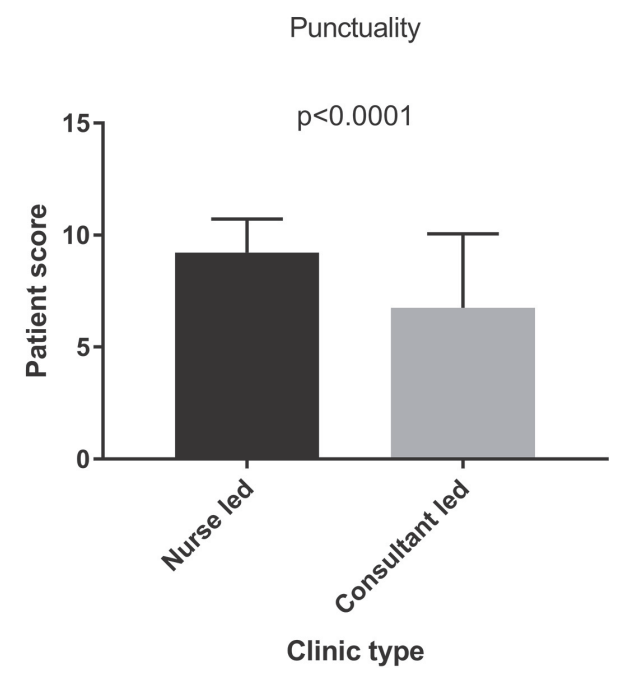

Figure 1. Patients visiting nurse-led clinics scored punctuality much higher than patients visiting consultant-led clinics.
Understanding of concerns

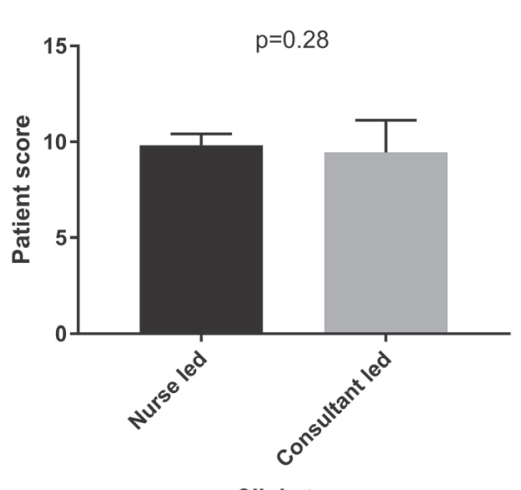

Clinic type

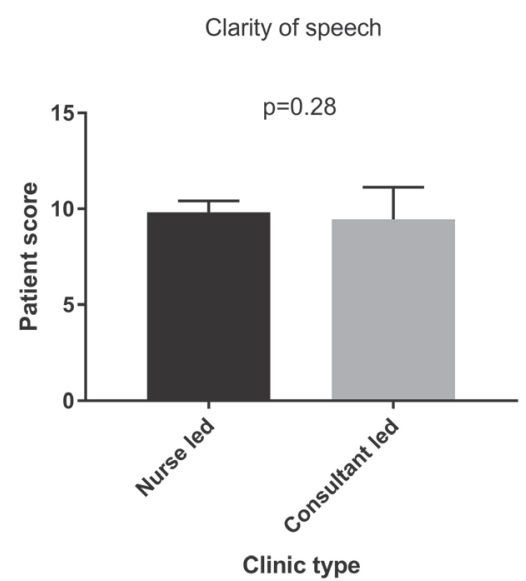

(c)

Figure 2. Patient scores for preparedness (a), understanding of concerns (b) and clarity of speech (c).

results demonstrate that the nurse-led clinic accomplishes similar outcomes to those achieved by the conventional doctor's clinic. However, the punctuality of nurse-led clinic was reported as significantly greater compared to the conventional clinic. This may be due to the fact that the more complex cases and the new cases were seen in the consultant-led clinic; whereas the nurses were only seeing the handpicked low-complexity routine follow-up cases.

The success of the nurse-led clinic depends upon the experience of the nurse and her clinical assessment skills. The patients who attend this clinic are highly vulnerable due to the nature of their disease and its progression and therefore clear communication between the nurse and the consultant is vital to make it successful without compromising the safety of the patients [15]. Our consultant had very good clinic guidelines and identified a patient population well in 


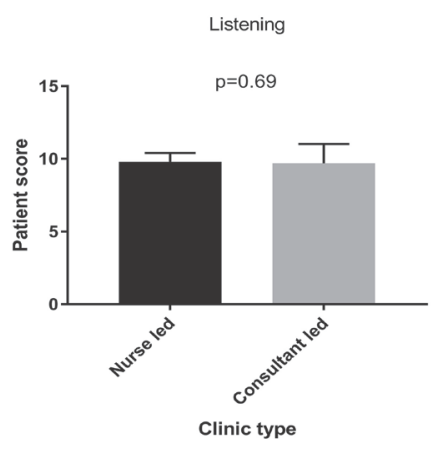

(a)

Explanation

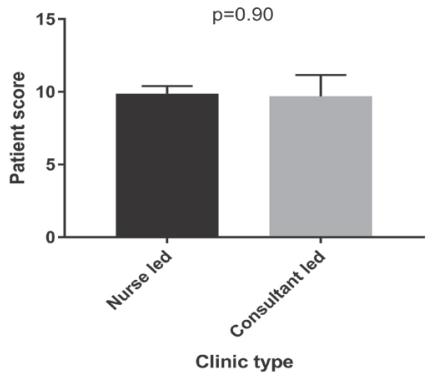

(c)

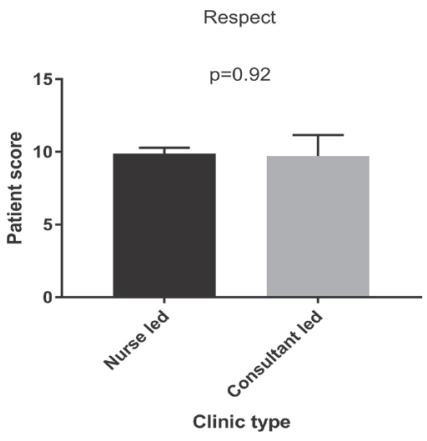

(b)

Allowing patients to speak

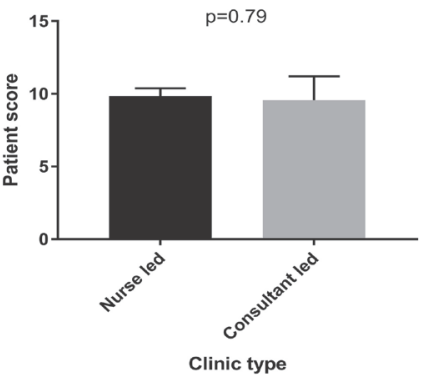

(d)

Figure 3. Patient reported scores for listening (a), respect (b), explanation (c) and allowing patients to speak $(\mathrm{d})$.

advance for the nurse-led clinic. They also had routine regular meetings to discuss patient progression. Recent trials have highlighted the efficacy of nurse-led clinics, with high patient satisfaction and high quality care [14] [16] [17]. The delivery of information and communication with the patients is also vital for the outpatient clinics in order to minimise the level of worry for the patients until their next clinic appointment. The provision of important information including what to do if there are any problems is key to making the clinic a success. Many studies in this area have demonstrated the importance of this as well as listening to patients' concerns and giving further advice when required [18].

We asked the patients to explain what elements of their clinic were useful. A more frequent comment from the nurse-led clinic was that lay language was often used, which was appreciated but was not always the case with the consultant-led clinics. The groups of patients we see in these cardiac departments tend to be elderly and may have cognitive impairments or hearing problems [19]. Ensuring that the information is provided in as clear manner as possible is important and will improve the relationship between service user and the health-care professional.

The nurse running the clinics was only committed to the outpatient duties. In contrast, the consultant has responsibilities for operating theatres and also other commitments in addition to the clinics. Our results support the concept that the nurse-led clinics are an efficacious way to reduce the workload of the consultants without impairing the quality of health care provision. Broers et al. (2006) have 


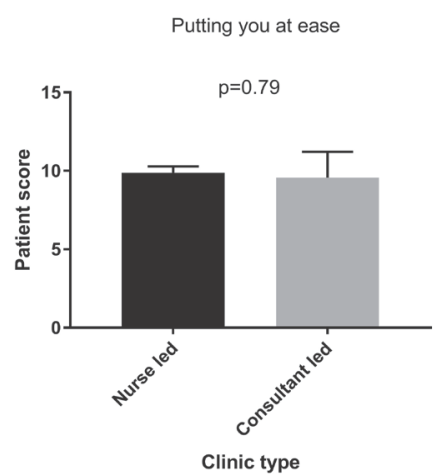

(a)

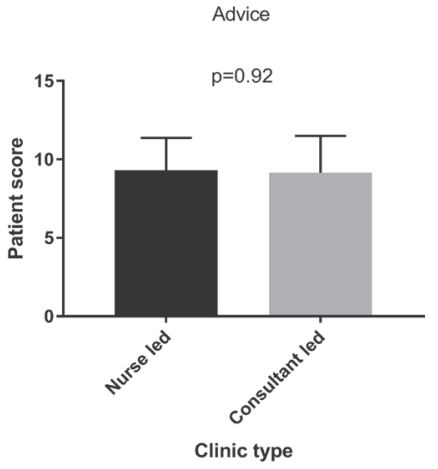

(c)

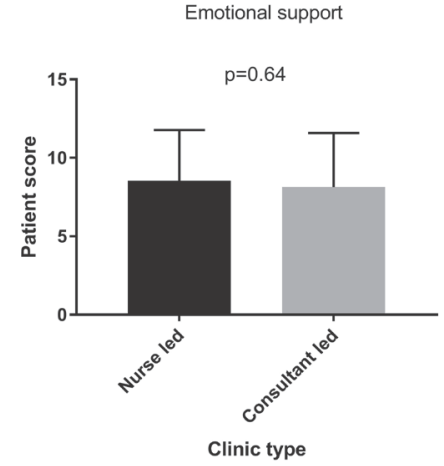

(b)

Advice for next follow-up

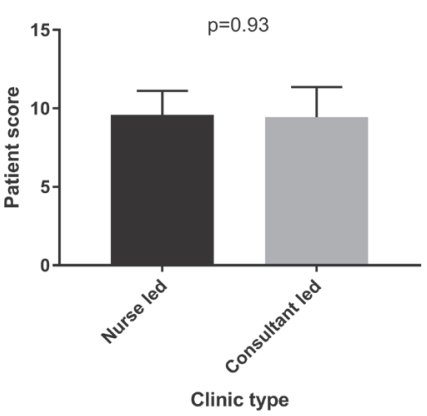

(d)

Figure 4. Patient reported scores for being put at ease (a), emotional support (b), advice (c), and advice for the next follow-up (d).

also shown that dedicated nurse practitioners can deliver a good quality nurse-led clinic with high patient satisfaction [14].

\section{Limitations}

The main limitation of this study was that the surgeon and nurse knew that an audit was being conducted. The results of our study may have impacted on the way they approached the patients during the study data collection period. The second limitation was that the patients seen by the nurse and consultants were different groups of patients and as such are independent of each other. Their individual requirements and preferences may have affected the comparability of the feedback between the groups.

\section{Conclusion}

In conclusion, a nurse-led routine follow-up clinic for patients with intrathoracic aortic dilatation can be safely and efficiently introduced in cardiac centres to reduce the outpatient waiting list and also improve punctuality of appointments compared to the consultant led-clinic. Clear guidelines must be established and followed, and only well described patient groups should be included. Efficient communication between the consultants and the nurses is crucial to ensure that the clinic is run safely without any hurdles. 


\section{Conflicts of Interest}

The authors declare no conflicts of interest regarding the publication of this paper.

\section{References}

[1] Williams, S., Williams, J., Tcherveniakov, P. and Milton, R. (2012) Impact of a Thoracic Nurse-Led Chest Drain Clinic on Patient Satisfaction. Interactive Cardiovascular and Thoracic Surgery, 14, 729-733. https://doi.org/10.1093/icvts/ivs056

[2] Hatchett, R. (2008) Nurse-Led Clinics: 10 Essential Steps to Setting up a Service. Nursing Times, 104, 62-64.

[3] Annandale, J. (2008) How a Nurse-Led Clinic Cut Outpatient Waiting Times. Nursing Times, 104, 45.

[4] Williams, D. (2006) Setting up a Cardiovascular Clinic in Primary Care. Nursing Standard, 20, 45-50. https://doi.org/10.7748/ns.20.43.45.s54

[5] Hill, J. (1997) Patient Satisfaction in a Nurse-Led Rheumatology Clinic. Journal of Advanced Nursing, 25, 347-354. https://doi.org/10.1046/j.1365-2648.1997.1997025347.x

[6] McMahon, R. (1998) Therapeutic Nursing: Theory, Issues and Practice. In: McMahon, R. and Pearson, A., Eds., Nursing as Therapy, 2nd Edition, Stanley Thornes, Cheltenham, 1-20.

[7] Sitzia, J. and Wood, N. (1997) Patient satisfaction: A Review of Issues and Concepts. Social Sciences and Medicine, 45, 1829-1843. https://doi.org/10.1016/S0277-9536(97)00128-7

[8] Husson, O., Mols, F. and van der Poll-Franse, L. (2011) The Relation between Information Provision and Health-Related Quality of Life, Anxiety and Depression among Cancer Survivors: A Systematic Review. Annals of Oncology, 22, 761-772. https://doi.org/10.1093/annonc/mdq413

[9] Davies, N.J., Kinman, G., Thomas, R.J. and Bailey, T. (2008) Information Satisfaction in Breast and Prostate Cancer Patients: Implications for Quality of Life. Psycho-Oncology, 17, 1048-1052. https://doi.org/10.1002/pon.1305

[10] Bergenmar, M., Nylén, U., Lidbrink, E., Bergh, J. and Brandberg, Y. (2006) Improvements in Patient Satisfaction at an Outpatient Clinic for Patients with Breast Cancer. Acta Oncologica, 45, 550-558. https://doi.org/10.1080/02841860500511239

[11] Reid, R., Haggerty, J. and McKendry, R. (2002) Defusing the Confusion: Concepts and Measures of Continuity of Healthcare. Final Report. Canadian Health Service Research Foundation, Ottawa.

[12] Nicol, N. (2000) Managing Atopic Dermatitis in Children and Adults. Nurse Practitioner, 25, 58-69. https://doi.org/10.1097/00006205-200025040-00004

[13] Smith, R. (1992) Audit and Research. British Medical Journal, 305, 905-906. https://doi.org/10.1136/bmj.305.6859.905

[14] Broers, C., Hogeling-Koopman, J., Burgersdijk, C., Cornel, J.H., van der Ploeg, J. and Umans, V.A. (2006) Safety and Efficacy of a Nurse-Led Clinic for Post-Operative Coronary Artery Bypass Grafting Patients. International Journal of Cardiology, 106, 111-115. https://doi.org/10.1016/j.ijcard.2005.03.009

[15] Hill, J., Bird, H.A., Harmer, R., Wright, V. and Lawton, C. (1994) An Evaluation of the Effectiveness, Safety and Acceptability of a Nurse Practitioner in a Rheumatology Outpatient Clinic. British Journal of Rheumatology, 33, 283-288. 
https://doi.org/10.1093/rheumatology/33.3.283

[16] Sakr, M., Angus, J., Perrin, J., Nixon, C., Nicholl, J. and Wardrope, J. (1999) Care of Minor Injuries by Emergency Nurse Practitioners or Junior Doctors: A Randomised Controlled trial. The Lancet, 354, 1321-1326.

https://doi.org/10.1016/S0140-6736(99)02447-2

[17] Mundinger, M.O., Kane, R.L., Lenz, E.R., Totten, A.M., Tsai, W.Y., Cleary, P.D., Friedewald, W.T., Siu, A.L. and Shelanski, M.L. (2000) Primary Care Outcomes in Patients Treated by Nurse Practitioners or Physicians: A Randomized Trial. JAMA, 283, 59-68. https://doi.org/10.1001/jama.283.1.59

[18] Clendon, J.M. (2003) Nurse-Managed Clinics: Issues in Evaluation. Journal of Advanced Nursing, 44, 558-565. https://doi.org/10.1046/j.0309-2402.2003.02845.x

[19] Wingfield, A. and Grossman, M. (2006) Language and the Aging Brain: Patterns of Neural Compensation Revealed by Functional Brain Imaging. Journal of Neurophysiology, 96, 2830-2839. https://doi.org/10.1152/jn.00628.2006 


\section{Appendix 1}

\section{UHSM \\ Your Hospital}

Thoracic aortic clinic-patient experience on nurse led compared to surgeon led clinic

Name of the patient:

Study Code:

Person seen the patient: Surgeon Yes -- No -- Nurse Yes --- No ---

1) Time of arrival to the clinic:

2) What time was your booked appointment?

3) Have you been seen on time for your booked appointment time? Yes --.---.-- No

If your answered no, how long did you have to wait? mins/hours.

4) Did the person speak clearly and understandable?

Yes -------- No

Any comments:

5) Did the person listen to you carefully and give you enough time to explain?

Yes

No

Any comments:

6) Did the person treat you with respect and dignity?

Yes --------- No -----------

Any comments:

7) Did the person explain things very clearly for your level of understanding?

Yes -------- No

Any comments

8) Did the person made you to feel at ease/comfortable?

Yes

No

Any comments:

9) Did the person fully understands your worries and concerns clearly and answered appropriately?

Yes --------- No -----------

Any comments: 
10) Did the person give you enough emotional support as you expected? Yes -------- No ---------

Any comments:

11) Did the person explain everything simple and concise as you expected?

Yes No

Any comments:

12) Did the person give you enough advice/support?

Yes No

Any comments:

13) Did the person explain clearly what would happen next? Yes No

Any comments:

14) How happy are you with your visit today?

On a scale of $0-100$ (please write down your number):

Many thanks for filling this form and your input is very valuable to us. 